\title{
Bericht zu: Le langage figuré - Colloque international en l'honneur du Professeur D. Dobrovolskij
}

\author{
Marine Espinat (Paris)
}

Im Juni 2013 fand in Paris ein internationales Kolloquium zu Ehren Dmitrij Dobrovol'skijs statt. Freunde aus der Wissenschaft und Freunde aus dem Leben versammelten sich, um den bedeutungsvollen wissenschaftlichen Beitrag des Jubilars zu würdigen und die Brücken, die sie durch seine Arbeit zu ihren eigenen Arbeiten schlagen konnten, vorzustellen. Dmitrij Dobrovol'skij ist zweifelsohne ein "Kopf voller Bilder", so stellte ihn Martine Dalmas vor. Und dennoch sei er, so bezeichnete er sich selbst, "kein Phraseologe, kein Germanist" und sei "überall" - wobei Ludmila Pöppel diese Aussage korrigierte: Dmitrij Dobrovol'skij ist nicht nur ein Phraseologe, nicht nur ein Germanist, sondern er ist überall und interessierte sich immer für alles.

Tatsächlich lässt sich sein Werk nicht auf Phraseologie und Germanistik beschränken, obwohl die Kombination dieses Bereichs und dieser Sprache sehr produktiv war und ihm internationale Anerkennung schenkte. Vielmehr ging es ihm darum, viele unterschiedliche Sprachen und deren Idiomatik aufzuspüren - und auf diesem Weg begleitete ihn oft Elisabeth Piirainen. Seine eigene Muttersprache, das Russische, untersuchte und untersucht er immer noch ergiebig mit Anatolij Baranov, Irina Levontina, Artem Šarandin, Natalia Ljubimova und Ludmila Pöppel. Deutschsprachige Germanisten verdanken ihm viele Denkanstöße und viele geöffnete Wege zum Weiterentdecken. Die Vielfalt der vertretenen Sprachen und Nationalitäten bei dieser Tagung ist repräsentativ für dieses "Überall-Sein" und seinen Wunsch nach wissenschaftlicher Kooperation. Moskau, Wien, Stockholm - und Paris... Er ist tatsächlich überall - und überall zu Hause.

\section{Kultursaliente Phänomene in der Phraseologie Dmitrij Dobrovol'skij (Moskau/Wien/Stockholm)}

Der Jubilar und berühmte Linguist Dmitrij Dobrovol'skij eröffnete den ersten Tag mit einem Vortrag über das Verhältnis von Phraseologie und Kultur. Als die Phraseologieforschung noch in den Kinderschuhen steckte und auch weit darüber hinaus, wurde behauptet, Phraseologie sei eine sprach- und/oder kulturspezifische Manifestation, die Erscheinung des Geistes einer Nation. Inzwischen konnte man etablieren, dass zahlreiche Idiome in verschiedenen europäischen Sprachen fast gleich vorkommen, obwohl die vollständige Äquivalenz nur selten gegeben ist. So schreibt man nicht "etwas schwarz auf weiß [haben/besitzen]" in gleicher Weise in Deutschland und in Russland. Idiome können nicht als Manifestationen des nationalen Geistes gelten, und dennoch spielt der kulturelle Bezug zu anderen semiotischen Systemen eine wichtige Rolle: Literatur, Kunst, Religion, Mythologie, Folklore (und noch viele andere kulturell besetzte Bereiche des alltäglichen Lebens) 
bereichern die Idiomatik der Sprachen. Insofern gehören diese Bezüge zum Inhalt der Idiome und sollten in den Wörterbüchern festgehalten werden. Eine solche kultursemiotische Betrachtung der Idiomatik deckt eine andere Definition der Kultur als "menschliche Kultur" und als Interaktion der Zeichen heterogener, aus ganz diversen Bereichen wie Literatur, Religion oder Folklore stammender Systeme auf.

\section{Inner form of idioms with magical force in Russian - best wishes and damnations Anatolij Baranov (Moskau)}

Der Vortrag Anatolij Baranovs über russische Idiome richtete sich an Dmitrij Dobrovol'skij selbst mit Wendungen des Erfolg- und Glückwünschens, aber auch an seine Feinde, mit Ausdrücken des Verdammens. Im Verzeichnis der dreizehn Idiome des Glückwünschens und der achtzehn Idiome des Verdammens wiederholen sich bestimmte Themen: Viele Glückwünsche basieren auf dem Religiösen und sind eher abstrakter Natur. Idiome des Verdammens sind dagegen viel häufiger konkreterer Natur, dies wird illustriert durch die starke obszöne oder körperliche Komponente einiger Idiome. Aufgrund dieser Eigenschaften verhalten sich die Idiome beider Gruppen jeweils anders: Bei den Idiomen des Glückwünschens lassen sich innere Form und aktuelle Bedeutung differenzieren, während dies für Idiome des Verdammens unmöglich ist.

\section{Wortverbindungsmuster und funktionale Chunks Katrin Steyer (Mannheim)}

Die Phraseologie besteht nicht nur aus idiomatischen Ausdrücken, sondern auch aus einer Vielfalt nicht-idiomatischer, fester und mehr oder weniger häufig vorkommender Wortverbindungen. Bei Syntagmen wie "ohne Vorliegen von Voraussetzungen" lässt sich Festigkeit in der lexikalischen und syntaktischen Struktur sowie in den Ko(n)texten ihres Auftretens feststellen. Bei der Analyse derartiger nicht-idiomatischen Syntagmen in Korpora stößt man wiederholt auf Muster verschiedener Typen. Solche Chunks sind nicht nur Strukturen mit Leerstellen zum Ausfüllen, sondern vielmehr vorstrukturierte Lösungen kommunikativer Aufgaben mit funktionalem Mehrwert. Die lexikalischen Füller der Leerstellen eines Chunks sind pragmatisch restringiert und alle Realisierungen dieser Chunks haben einen gemeinsamen Nenner auf der Ebene der abstrakten Bedeutung. Die korpusgesteuerte Untersuchung solcher Strukturen und deren Rekurrenz legt die Vermutung nahe, dass diese Ausdrücke als einzigartige unikale Wortschatzeinheiten abgespeichert sind. $\mathrm{Zu}$ den Desiderata der Forschung gehört nach einer solchen Erkenntnis auch die Einführung neuartiger lexikografischer Aufbereitungen und Darstellungen in Form syntagmatischer Musterwörterbücher.

\section{$4 \quad$ Figurative Language und Konstruktionsgrammatik (sprach)historisch betrachtet Natalia Filatkina (Trier)}

Der Beitrag einer (sprach)historischen Betrachtung ist aufschlussreich, was den Prozess der Idiomatisierung, der Entstehung des sprachlichen Bildes und der Konventionalisierung betrifft. Diese historische Perspektive ist notwendig, was anhand von drei Aspekten verdeutlicht wird. Zum ersten: was synchron betrachtet als "Endprodukt" in sehr fester lexikalisierter Form erscheint, ist eigentlich das Resultat der diachronen construction paths. Zweitens kann man feststellen, dass die Variation, die zur Konventionalisierung führt, noch 
vieler Erklärungen bedarf. Letzten Endes muss auch der Wandel des mentalen Bildes in Betracht gezogen werden. Dieser Studie widmete sich die Nachwuchsforschergruppe HiFos der Universität Trier. In dieser Hinsicht erinnerte der Vortrag von Frau Natalia Filatkina jeden Phraseologen der Synchronie daran, dass die drei definitorischen Bausteine der Phraseologie (Polylexikalität, Festigkeit und Idiomatizität) in einer diachronen Perspektive keine Selbstverständlichkeit sind, und dass jedes Idiom seine Geschichte hat, die nicht ausgeklammert werden darf.

\section{$5 \quad$ Flüchtige Figuriertheit Annette Sabban (Hildesheim)}

Idiome charakterisieren sich besonders durch Festigkeit und ihre Bildkomponente. Jedoch kann die Festigkeit flüchtig werden, indem die Idiome nicht in ihrer prototypischen Form übernommen, sondern der Sprecherfantasie nach angepasst werden. Anhand von Korpusbelegen diverser Natur (journalistische Texte, gesprochene Sprache, usw.) wurde gezeigt, wie die Sprachbilder innerhalb eines Szenarios ausgebaut werden, um sich dem Ko(n)text anzupassen. Darüber hinaus können Sprachbilder wie Fäden weiter gesponnen und zum Spiegel der Sprecherkreativität werden. Schließlich können sie zu Neukombinationen mit anderen Metaphern umgearbeitet werden. Dabei reicht die Notion des Mappings nicht aus, um solche Erscheinungen vollständig zu erklären. Diese Produktionen illustrieren die Freiheit der Sprecher, nicht systematisch auf einen Quellbereich zurückzugreifen, nicht unbedingt alle Komponenten einer Metapher zu nutzen und sich auch nicht unbedingt an ein Standardszenario zu halten. Die Fantasie hat das Wort.

\section{Besonderheiten diachroner Metaphern und ihre Übersetzungen - Am Beispiel der Hofwechselstrophe Walthers von der Vogelweide Artem Šarandin (Moskau/Wien/Stockholm)}

Die Konventionalisierung einer Metapher ist ein historischer Prozess, deren Rekonstruktion heute teilweise schwer erfassbar ist. Einige Metaphern etablieren sich, andere sterben aus, weshalb man ihnen nur in alten Texten auf die Spur kommen kann. So untersuchte Artem Šarandin metaphorische Okkurrenzen des Kranichs und des Pfaus in Hofwechselstrophen Walthers von der Vogelweide: "dô fuort er mînen krenechen trit in die erde, dô gienc ich slîchent als ein pfawe". Über das Interesse an verstorbenen Metaphern hinaus, stellt sich die Frage ihrer Übersetzung, die meistens nur wörtlich erfolgt - was das Verständnis des heutigen Lesers sicherlich deutlich erschwert. Wer ist noch im Stande, das Bild des Kranichs mit Ordnung, einer ritterlichen Figur oder Freiheit zu verbinden und das des Pfaus mit Schönheit, Eitelkeit und dadurch mit dem Geistlichen/Religiösen? Die Konfrontation mit derartigen Texten und Metaphern machen dem Linguisten und dem Übersetzer bewusst, dass er auf seine synchrone Perspektive verzichten muss, um mittelalterliche Texte $\mathrm{zu}$ analysieren und ihre Bedeutung angemessen wiederzugeben.

\section{$7 \quad$ Semantische Präferenzen bei der Phrasembildung aus kontrastiver Sicht Gottfried Marschall (Paris)}

Die Bilder der Sprache spiegeln Repräsentationen wider, etablieren sich in einer Sprachgemeinschaft und beeinflussen unsere Welterfahrung. Im Sprachalltag kann man feststellen, dass bestimmte semantische Felder besonders intensiv für die 
Phraseologismenbildung genutzt werden und anhand einer kontrastiven Analyse ließen sich bestimmt kulturelle Unterschiede feststellen: werden die Unterschiede der Idiomatik größer, je weiter die Sprachgemeinschaften voneinander entfernt liegen? Frankreich und Deutschland teilen einen großen Teil ihres Kulturguts und dennoch sind Unterschiede zu bemerken, wie zum Beispiel die Vorliebe der Deutschen für das Essen und der Franzosen für das Trinken. Auch was die Kunst betrifft, unterscheiden sich beide Länder und Sprachgemeinschaften: Deutsche gelten als musikaffin, Franzosen als Liebhaber der Malerei. Hinweise auf derartige nationale Neigungen lassen sich in der Idiomatik der jeweiligen Sprache wiederfinden.

\section{$8 \quad$ Zum alltäglichen Spiel mit sprachlichen Bildern Elisabeth Piirainen (Münster)}

Im alläglichen Leben sind wir umgeben von Bildern, darunter auch sprachlichen, deren Gebrauch in der Werbung und in der Presse stetig zunimmt. Oft werden diese sprachlichen Bilder anhand von Sprachspielen aktualisiert, beruhend auf der Veranlagung des Homo ludens und dem Ambiguitätspotential der Sprache. Typographie, Homophonie, Assonanzen, Wortbildung und Phraseologie gelten als bevorzugte Mittel des Sprachspiels. Auch dessen Funktionen sind vielfältig: das Schließen einer Benennungslücke auf spielerische Art, Selbstzweck, Überraschungseffekt und/oder die Suche nach Wirkung einer besonders starken gesellschaftspolitischen Aussage. Fraglich ist aber noch, inwiefern diese Sprachspiele ihr Ziel erreichen: Kaufen Kunden mehr? Werden die Rezipienten dadurch empfänglicher? Sind viele Sprachspiele nicht an den Haaren herbeigezogen oder zu komplex, um noch planmäßig wirken zu können? Oder finden etwa die Linguisten immer ein Haar in der Suppe?

\section{9 Über Gedankenblitze und Fallstricke: Eine Einzelfallanalyse der Verarbeitung figurativer Sprache Michael Schecker (Freiburg i. Br.)}

Seit der kognitiven Wende der Linguistik und der Phraseologie ergaben sich viele Fragestellungen, die lange ungeklärt blieben. Dank neurolinguistischer Untersuchungen gewinnt man endlich einen Einblick in die Verarbeitung der figurativen Sprache. Lange blieb umstritten, ob die figurative Bedeutung von der wörtlichen Bedeutung aus erarbeitet werden müsse oder ob figurative und wörtliche Bedeutungen unabhängig gespeichert und somit unabhängig abrufbar seien. Inzwischen wurde nachgewiesen, dass eine wörtliche Verarbeitung bei jedem figurativem Ausdruck stattfindet, sogar bei Ausdrücken, die gar nicht wörtlich verstanden werden können. Die Erscheinung eines metaphorischen Ausdrucks verursacht eine deutliche Reaktion (im N400 Bereich), die als Signal für die Aktivierung anderer Ressourcen gilt. Ob diese Reaktion durch Inkohärenz ausgelöst wird, bleibt fraglich. Interessant ist dabei die Tatsache, dass die N400-Reaktion nicht nur in der linken für Sprachverarbeitung zuständigen Hemisphäre erfolgt, sondern auch in der rechten, was darauf hindeutet, dass es bei figurativen Ausdrücken zu einem Kontextabgleich kommt. Wichtig ist auch, dass all diese Prozesse so schnell ablaufen, dass die unterschiedlichen Verarbeitungsschritte gar nicht bewusst werden können und wir somit nicht den Kopf verlieren. 


\section{0 "Quotation" Prosody in Russian}

\section{Irina Levontina (Moskau)}

Die Redewiedergabe wurde seit der Studie Jakobsons über shifters zum Gegenstand vieler linguistischer Untersuchungen. Die Sprachen verfügen über sehr unterschiedliche Mittel der Redewiedergabe, die im Rahmen der evidentiality theory beschrieben und analysiert wurden, aber immer ist die Unterscheidung zwischen der eigenen Äußerung und der des Anderen entscheidend. Im Russischen erfolgt die Redewiedergabe oft durch zwei bestimmte Partikeln (Вот und Ax), die beide die Redewiedergabe einleiten und dennoch anders benutzt werden. Die erste dient der Wiedergabe kürzerer, direkt zitierter Ausschnitte, die zweite der Erinnerung an eine erwähnte Kernausgabe. Dabei darf die Intonation nicht vergessen werden, die dem Hörer signalisiert, dass der Sprecher hier die Worte einer anderen Person wiedergibt. Dieses letzte Mittel ist bestimmt das flüchtigste und dennoch bleibt es das signifikanteste, da die russische Sprache über keine grammatikalisierten Bedeutungen der Redewiedergabe verfügt.

\section{Figuriertheit im Weindiskurs \\ Laurent Gautier (Dijon)}

Der Weindiskurs hat eine eigene Idiomatik, die die Geschmacksdeskriptoren in exotische Benennungen verwandelt. Laurent Gautier ging es darum, dieser Figuriertheit auf die Spur zu kommen, um herauszufinden, welche Transfermechanismen ihr zugrunde liegen. Anhand eines Korpus, bestehend aus Aufnahmen von Kommentaren bei Weinproben (mündlich) und schriftlichen Beschreibungen von Weinen, sowohl von Laien, als auch von Experten, wurde der Begriff der Mineralität des Weins analysiert. Immer wieder hört man, dieser Wein sei besonders mineralisch. Handelt es sich dabei um einen Expertenterminus, den die Laien versuchen sich anzueignen? Oder bleibt die Bezeichnung für jeden, ob Experte oder Laie, unklar? Es stellt sich heraus, dass es sich tatsächlich zum größten Teil um eine gescheiterte Terminologisierung handelt, und dass Mineralität keinen messbaren Wert darstellt und sehr approximativ mit dem Begriff des Terroirs in Verbindung gebracht wird, der die Eigenschaften des Herkunftsgebiets bezeichnen soll.

\section{Phraseologie in der politischen Küche Natalia Ljubimova (Moskau)}

Die deutsche Vorliebe für Wurst illustriert sich vielfältig - wie z. B. in die Initiative ProBockwurst zur Anerkennung der Bockwurst als Kulturgut. Und diese Vorliebe spiegelt sich in der Idiomatik der Sprache - wozu die Phraseologen ihren Senf zu geben haben. Es stellt sich heraus, dass das Wurst-Bild sehr produktiv ist, unter anderem weil viel mit Doppelsinn und kompatiblen gastronomischen Bildern gespielt wird. Allgemeiner scheinen gastronomische Metaphern und Ausdrücke sehr beliebt. Eine Kampagne für die Nationalratswahlen in der Schweiz nahm Gastronomie als Grundmetapher der ganzen Kampagne: jede Partei durfte sein eigenen Zutaten zum Gelingen des "Rezepts", also ihres politischen Programms in appetitlicher Form vorstellen. Somit wird eine Verbindung zwischen Küche und Politik hergestellt: beide erfordern Aktivität, Verantwortungsbewusstsein und Gelingen des Resultats. Ob man damit den Wählern die Kampagne schmackhaft macht? 


\section{Phraseology between the two Russian revolutions in Pravda 1917 Ludmila Pöppel (Stockholm)}

Die Pravda gehörte zu den für die sowjetische Zeit signifikantesten Zeitungen in Russland, denn sie blieb über siebzig Jahre lang das Sprachrohr des Regimes. Allerdings war sie zwischen beiden russischen Revolutionen (Februar- und Oktoberrevolution des Jahres 1917) nur eine Zeitung unter zweitausend anderen, in dieser instabilen, von Machtkämpfen geprägten Periode, in welcher der russisch-sowjetische politische Diskurs entstand und sich verbreitete. Anhand dieses journalistischen Korpus ging es Frau Pöppel darum, die von der Phraseologie widergespiegelten konzeptuell-semantischen Netze ans Licht zu bringen. Idiome sind in solchen Texten gute Illustrationen des gängigen politischen Diskurses. Die Taxonomie der häufig vorkommenden Konzepte (alt/neu) und Lemmata (z. B. Krieg) zeigte sich tatsächlich repräsentativ für den Geist dieser Zeit. 